\title{
Big Data in the City
}

2015 Big Data Challenge for High School Students:

Conference Proceedings Abstracts

by Emma Adamson-De Luca, Silsopang Paknara, Tasneem Badshah, Maxim Vorobyov, Adam Kline, Anam Qureshi, Tuleepraj Thiyagarajah, Pricemar Chevelon, Tasha Reece, Kamran Salehi, Garrett Singh, Hayden Tran, Taqwa Alnoor, Hera Khan, Jessica Kumaran, Saliem Talash, Anupya Pamidimukkala, Fei Dong, Jessica Ip, Pamela Zeng, Kevin Leung, Jerry lu, Gabriel Gelgor, Arbri Halili, Arian Mohamad-Hosaini, Daniel Yu, Raj Prakash. Daniel Rubinstein, Michael Schmittat, William Kwong, Thomas Beckley, Daniel Weisdorf, Claire Bettio, Joseph D'Ercole, Thomas Lawrynuik, Alexandra Leone, Jenny Baek, Evgeny Bogopolskiy, Justin Palombo, Alan Zhang, Johnathon Martin, Valerie Hermanns, Lisa Taibi, Anthony Cavallin, Tommy Xiang, Allen Bai, Steffen Cucos

\section{Disclaimer}

These abstracts are provided for all student teams that have submitted project reports by December 20, 2015. The STEM Fellowship Journal editorial board has made every effort to ensure proof and English editing of these abstracts in a limited amount of time, and neither organization as a whole or any of its volunteer members can be held accountable for inaccuracies that may have occurred in the abstract publication. Abstracts are published in alphabetical order per school names of the participating teams.

\section{Big Data 2015: Teen Pregnancy}

Emma Adamson-De Luca, Silsopang Paknara,

Tasneem Badshah

The Abelard School

\section{Abstract}

Using big data and statistical modeling tools such as $\mathrm{R} \mathrm{cmdr}$, we were able to trace teen pregnancy to a seemingly unlikely cause: unemployment. This may have not been the most obvious insight to the teen pregnancy issue when we began this project, but by probing the data we were able to find this unexpected answer. Big Data, when coupled with powerful analysis tools, is of immense worth in converting raw data into powerful insights. This is why the Open Data Initiative by the City of Toronto is so important.

\section{Key words}

Teen Pregnancy, R-language Modelling, Unemployment

\section{Big Data in the City with a Focus on Socioeconomic and Ecological Enviroment \\ Maxim Vorobyov, Adam Kline \\ The Abelard School}

\section{Abstract}

We decided to take a careful look into two most important characteristics of our city -people and the environment, and study how they are connected to each other. To do so, we set up two independent sets of variables and then created two rankings for each neighborhood based on these two dimensions: human and environmental, in order to compare the relation between the two.

Environmental Index are not at all correlated between themselves. Also, they do not make up an equal share of share of the Neighborhood Index, which once again, disproves the hypothesis. This may be affected by the fact that there were 8 socioeconomic factors and only 4 environmental ones, but nevertheless, the difference should not be so overwhelming.

From these conclusions, the main factors affecting these indexes are Percent of Workforce Unemployed, Percent of Recent Immigrants Relative to Total population and Percent of Population in Labor force. These are the main areas the city of Toronto should focus on and ameliorate, to improve the life quality of the people living in the city.

\section{Key words}

Big Data, Toronto, People, Environment 


\section{High School Dropout Rates}

Anam Qureshi, Tuleepraj Thiyagarajah, Pricemar Chevelon

David and Mary Thomson Collegiate Institute

\section{Abstract}

This research is done to elaborate upon geographic areas and number of dropouts in high school in Toronto. The goal is to find the correlation between geographic areas and number of dropouts in high school, and if there are any increases or decreases in the graduation rate of high school students. There have been dropouts over five years from 2006-2011. There were 18,265 students in the TDSB on October 31, 2006 who were between 13 and 15 years of age and who were new to secondary school. Their progress was closely studied over the five years. TDSB lost 1,900 students in that time. The approach was to find the reason why students dropout from high school.

\section{Keywords}

Number of Dropouts, High School Students, TDSB, Geographic Areas, Big Data

\section{Toronto's Bike Lanes - A Change to the Way we Move Tasha Reece, Kamran Salehi, Garrett Singh, and Hayden Tran David and Mary Thomson Collegiate Institute}

\section{Abstract}

The population of Toronto grows day by day. The major problems of traffic, accidents and fatalities caused are also growing rapidly. By collecting and analyzing the data related to transportation methods used by individuals in the Greater Toronto Area, it has been found that in comparison to many of the other cities with fast growing populations, it is easily noticeable that Toronto is limited when it comes to the availability of bike lanes.

This limitation is causing a decrease in the number of individuals that use bicycles as one of their main method of transportation. If more citizens within Toronto were capable of travelling from one point to another using a bicycle, the traffic and accidents within Toronto would significantly decrease. While analyzing this data, several other problems were noticed with regards to the safety of the already available bike lanes in Toronto.

Some areas which contain bike lanes have provided cyclists with limited space to a point where the life of the cyclists are at risk and many collisions are happening in these lanes. Increasing bike lanes in Toronto is a very efficient way to take a step towards solving the problem of traffic and collisions in Toronto and by providing cyclists with more space in their lanes.

\section{Keywords}

Bike Lanes, Bike Toronto, Transportation Methods, Bike Accidents

\section{Post-Secondary Education}

Taqwa Alnoor, Hera Khan, Jessica Kumaran \& Saliem Talash David \& Mary Thomson C.I

\section{Abstract}

The purpose of this research is to determine the employment rates after post-secondary

education. The approach was to determine the average of how many people get jobs after graduating college vs. graduating university and then to compare them to each other. The hypothesis was that the graduate employment rates for colleges would be higher than universities. Our research showcased that the graduate employment rates of universities was higher than colleges.

\section{Keywords}

Big Data, College, University, Employment Rates, Success or No Success 


\section{Diving into Debt: a Study on Factors Affecting Debt Risk Score in Toronto}

\section{Anupya Pamidimukkala, Fei Dong, Jessica Ip, Pamela Zeng}

Earl Haig Secondary School

\section{Abstract}

This research paper aimed to find correlation between different data found in the 140 Toronto neighborhoods and debt risk. Debt risk was compared with select variables from available data, including education, health, environment, housing, economics, demographics, transportation, recreation, and safety. The purpose of this study was to help civilians as well as the government to identify possible factors that lead to higher debt risk, as well as find solutions to reduce it.

The data was retrieved from Open Data Toronto. The program used to analyze the data was SAS Studio. A linear regression model was built to determine the factors that have a seemingly great correlation with debt risk. It was concluded that the percentage of people who receive social assistance, the percentage of people who applied for rent banks, and the number of reported sexual assaults in a neighborhood had a positive relationship with increased debt risk. The age-adjusted rate of people who received breast cancer screening had a negative correlation with increased debt risk.

Through the results, several solutions can be proposed to reduce debt risk. More education on safety and health can lead to citizens becoming more responsible and aware of their financial troubles. Giving other forms of aid that are not monetary may be beneficial to help people get out of debt and become financially independent.

\section{Keywords}

Big Data, Debt Risk Analysis, Social Assistance, Toronto, Factors Affecting Debt Risk

\section{Collision Statistics: a Study in Toronto Road Safety Kevin Leung, Jerry Iu, Gabriel Gelgor and Arbri Halili Earl Haig Secondary School}

\section{Abstract}

For the Big Data Challenge, we have set out to determine the major causes of vehicle collisions in the City of Toronto, and propose solutions to this issue. We have made use of Toronto Open Data to gather statistics on wellbeing demographics, traffic, economics, and collisions. We analyzed the data and determined six neighbourhoods that deviated from the normal ratio of collisions to road volume. We researched these six neighbourhoods and determined that most accidents occur in commercial areas and the least accidents happen in residential areas. Residential areas are not areas where large amounts of people collect daily, but commercial areas are. Commercial areas also have a lot of pedestrian movement, and coupled with the high number of vehicles makes for many collisions. Vehicle accidents can be reduced in a few ways, such as producing more PSAs, enforcing jaywalk prevention, reducing speeding, and employing advents in driving technology such as driverless cars.

\section{Keywords}

Commute, Neighbourhoods, Accidents, Safety, Toronto

\section{Data Analysis: Urban Area and Air Pollution Arian Mohamad-Hosaini, Daniel Yu Earl Haig Secondary School}

\section{Abstract}

This is a research and analysis big data project by studying Toronto's environmental condition and air pollution. Based on the dataset of amount of air pollution and related information within 140 Toronto neighborhoods, we use python and internet resources to investigate the correlation between these data. The purpose of this project is to collect data on the pollutant score from non-industrial area in Toronto, arrange and organize it for the purpose of analyzing factors related to environmental protection. Analysis done by comparing area of green space and traffic condition with relation to pollutants in a neighborhood and compare data between neighborhoods. The significance of this project is that big data may help 
come up with solutions to environmental issues such as pollution, climate change, and waste. As big data on environment may have a bigger role with resource management or environment monitoring in the future.

\section{Keywords}

Air pollution, Environment

\section{How Green Does Toronto Tweet?}

Raj Prakash. Daniel Rubinstein, Michael Schmittat The Academy for Gifted Children - P.A.C.E

\section{Abstract}

Only recently has social media become a large part of our daily lives. With this comes the amazing ability to easily spread and publicize information. Our goal within this paper is to attempt to understand whether social media is an accurate representation of real life activity. In order to do so, we are going to look at various Toronto "green" related Twitter trends and compare their popularities to that of some real life initiatives. By using online resources as well as writing graphing and text editing code, we have been able to show the differences amongst these trends. Our results showed that the popularities of Twitter green (eco) trends differed from real life indicators. This information allowed us to conclude that our online activity in no way accurately represents our real world initiatives.

\section{Keywords}

Twitter, Tweet, Green, Data Set, Toronto

\section{Toronto: Correlation in Big Data, Science, and Environmental Issues \\ William Kwong, Thomas Beckley, Daniel Weisdorf \\ The Academy for Gifted Children - P.A.C.E.}

\section{Abstract}

Toronto is a city that prides itself on a multitude of aspects, such as its cultural diversity and its consistent rating as one of the most livable cities in the world by the Mercer Quality of Living Survey. Of course with any cities come flaws and issues it must tackle. Currently, the issue of the environment and developing Toronto into an environmentally sustainable city is one sought to be resolved. The rapid expansion and industrialization of the world has created issues that must be solved quickly. Governments no longer seek to exploit the environment, they seek to repair the environment. However, information must be gathered and analyzed before action is taken. Big Data is a term used to describe data sets that are so large and complex that it renders traditional methods of data processing applications inadequate. Challenges that arise with Big Data include the analysis, capture, and visualization of large data sets. In order for information to be interpreted and used effectively, Big Data must be condensed and made palatable. In Toronto, data is collected daily on weather conditions, air pollutants, and other environmental factors. With datasets being updated daily, the once small, gentle giants become a mass of rock. Rock that we must chisel away to reveal the bigger picture. The use of statistical analysis in the form of $R$ enabled the gathering of information from datasets containing data on Torontonian neighborhoods pertaining to green energy installations and environment conditions. Using the information gathered, the neighborhoods of Toronto were scored by their commitment to Toronto's environment policies and how 'green' they were objectively. Toronto as a whole has a long way to go before it becomes the utopia it strives to be, but improvements such as the sole use of renewable energy and strict environmental regulations will help Toronto become that utopia.

\section{Keywords}

Data analysis, renewable energy, Toronto 


\section{Bettering Life in Toronto One Cluster at a Time} Claire Bettio, Joseph D'Ercole, Thomas Lawrynuik, and Alexandra Leone Villanova College

\section{Abstract}

As the world develops and the population grows at a rapidly increasing rate it has become progressively more difficult to plan the future necessities of cities and communities. A relatively new field, with applications in community planning, is the analysis of big data Given a large set of demographic data, the task was to develop a means of analyzing the data to benefit life, work, and/or play in Toronto's neighbourhoods. Using Big Data analytics, the needs of certain areas were predicted, whether that be an aging population that requires increased assisted living programs or communities in need of education retention strategies. To accomplish this, five major programs were written in Python (Sorter.py, SDCalculator.py, Clustermaker.py, Clusterprinter.py, and Comparisonrunner.py). The Sorter.py and SDCalculator.py programs first sort the data into a more usable form, then calculate standard deviation. The Clustermaker.py program sorts and groups the given data from each geographic region into eight distinct categories, and six clusters within those eight categories. Clusterprinter.py prints a chart to excel to display the clusters, and Comparisonrunner.py searches for correlations present between two clusters. Out of an intended 48 clusters, 4 clusters had no neighbourhoods satisfying the criteria, so only 44 clusters were calculated and were available for analysis. These clusters define how far from the average (for each specific category analyzed) any neighbourhood may be according to standard deviations (this is according the percent form for each category relative to total population, excluding population density and average family income). From these clusters, comparisons were done between subgroups to determine if there were any correlations in the data. In general, this program was created as a tool that can be used to cluster big data, ultimately allowing an analyst to easily draw conclusions on how to appropriately plan the future of communities, and therefore increase the quality of life in Toronto.

\section{Keywords}

Big Data, Predicting, Python, Clustering, Demographic Analysis, Community Planning

\section{Environmental Analysis of Toronto Neighborhoods Jenny Baek, Evgeny Bogopolskiy, Justin Palombo, and Alan Zhang Villanova College}

\section{Abstract}

Improving the environment is always the responsibility of the government, which has been trying to set up green rebate programs, install renewable energy projects, and reduce the pollutants released into our environment. Obviously, it is wise to put more effort into those neighborhoods with the most serious environmental conditions. To help the government determine the focus of its work with the environment, we took some data about environmental conditions and renewable energy projects in Toronto, formatted it, classified it, aggregated it, and analyzed it with Python. Reading data of any year's environmental condition, our program can output the neighborhoods with the worst environmental conditions and some noticeable indexes which may give users valuable suggestions.

\section{Keywords}

Environmental Analysis, Toronto Neighborhoods, Big Data, Python

\section{Best Areas in Toronto for Immigrants to Settle Johnathon Martin, Valerie Hermanns, Lisa Taibi, and Anthony Cavallin \\ Villanova College}

\section{Abstract}

For a high school competition we were given a large amount of municipal data for the city of Toronto and we were asked to use it to discover new ideas about life, work, and play in the city. Based upon this we decided to use this data to determine which part of the city is best suited to new immigrants. Using these results we can aid in better assimilating new immigrants into Toronto life, as well as insuring that they have similarities with their neighbours therefore limiting the stress put on the community as a whole. The city is split into 44 wards which we used to break the city into parts. We evaluated data from each ward such as the average income, ethno cultural data, commonly spoken languages, and number of recent 
immigrants (from each part of the world). We then compared the variables listed above of each ward to the unique circumstances of each immigrant, effectively evaluating which wards would be best suited for them to settle in. To accomplish this we first started by analyzing the data using Microsoft Excel, and then we created a program which compared the data for each ward to the circumstances of each immigrant and output the wards best suited to that immigrant.

\section{Keywords}

Data analysis, Toronto, immigration, settlement

\section{Relevance of Emergency Requests and Emergency Station Placement}

Tommy Xiang, Allen Bai, and Steffen Cucos

York Mills Collegiate Institute

\section{Abstract}

This project was done for the Big Data Challenge hosted by the STEM Fellowship by our group, consisting of three high school students. We decided to use data sets on Toronto's safety statistics, which included the number of accidents, ambulance calls and crimes, to produce maps of Toronto that are colour coded in order to show "hot spots" in terms of calls to emergency services. The goal of this was to see if there is existed disproportionately high amounts of requests in some areas compared to the average, and to see if these areas were in fact further away from first responders than others. By mapping the data to a map of Toronto neighborhoods, overlaying the locations of first responders, and finally calculating the average distance to nearest emergency service stations for each neighborhood, we were able to show that there are in fact neighborhoods in Toronto that can be considered to be hotspots for emergency requests, and are further away or similarly distanced from first responders than other neighborhoods with lower accident counts.

\section{Key words}

Emergency, Location, Visualization, Data mapping
The STEM Fellowship 2015-2016 Big Data Challenge is a multidisciplinary competition that enables high school students to mine open-source data and use it to analyse socioeconomic and environmental issues. The participants expertly used various statistical analysis tools such as Python's Pandas analysis library, R, Dell Statistica and SAS Studio. This year's challenge was organized around open data available on the city of Toronto in Ontario, Canada.

Published here are students' project abstracts. The research topics include data analyses of important transit issues such as bike lanes and vehicle collisions, education issues, employment dynamics connected to post-secondary education, and high school dropout rates. Other projects analysed the city's environment by various factors in an effort to evaluate air pollution and impacts of green energy installations. The research presented by this year's participants indicates a use of insight and creativity as they explored a multitude of different aspects of life in the city.

On behalf of STEM Fellowship, I congratulate all students who participated in the challenge. I believe they will continue their work with Big Data in various research fields with excellence.

We look forward to future Big Data Challenges expanding across Canada and providing even more students with a challenge to research their cities through Big Data.

\section{Nikki Sigurdson \\ Operations Director, STEM Fellowship \\ Big Data Challenge 2015-2016 Team Lead}

